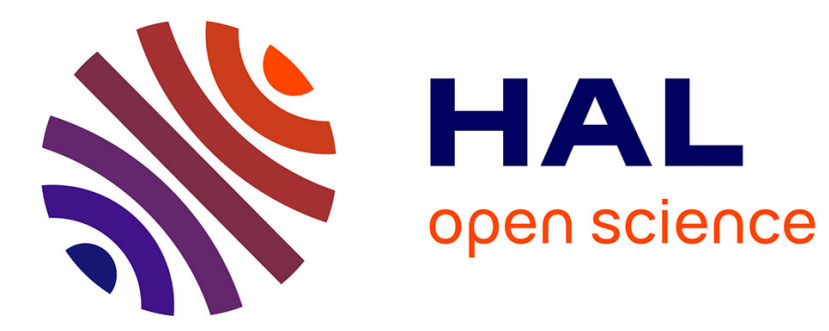

\title{
Dispositif pour la mesure de l'absorption optique a 10,6 $\mu \mathbf{m}$ \\ D. Bois
}

\section{To cite this version:}

D. Bois. Dispositif pour la mesure de l'absorption optique a 10,6 $\mu \mathrm{m}$. Revue de Physique Appliquée, 1976, 11 (2), pp.293-298. 10.1051/rphysap:01976001102029300 . jpa-00244060

\section{HAL Id: jpa-00244060 https://hal.science/jpa-00244060}

Submitted on 1 Jan 1976

HAL is a multi-disciplinary open access archive for the deposit and dissemination of scientific research documents, whether they are published or not. The documents may come from teaching and research institutions in France or abroad, or from public or private research centers.
L'archive ouverte pluridisciplinaire HAL, est destinée au dépôt et à la diffusion de documents scientifiques de niveau recherche, publiés ou non, émanant des établissements d'enseignement et de recherche français ou étrangers, des laboratoires publics ou privés. 


\author{
Classification \\ Physics Abstracts \\ $0.640-2.410-2.632-8.812$
}

\title{
DISPOSITIF POUR LA MESURE DE L'ABSORPTION OPTIQUE A $10,6 \mu \mathrm{m}\left({ }^{*}\right)$
}

\author{
D. BOIS \\ L. E. P., 3, avenue Descartes, 94450 Limeil-Brévannes, France
}

(Reçu le 16 juin 1975, accepté le 22 octobre 1975)

\begin{abstract}
Résumé. - Nous décrivons un dispositif destiné à mesurer de faibles coefficients d'absorption par une méthode calorimétrique. L'appareil réalisé est un calorimètre à conduction, dont les fluxmètres thermiques sont constitués d'éléments à effet Peltier semiconducteurs ; il permet d'effectuer la correction de lumière diffusée. Sa sensibilité est d'environ $10^{-5} \mathrm{~cm}^{-1}$ et la précision meilleure que $10 \%$ autour de $10^{-2} \mathrm{~cm}^{-1}$. Nous donnons quelques résultats obtenus sur $\mathrm{GaAs}, \mathrm{Ge}, \mathrm{NaCl}$ et $\mathrm{KBr}$ à $10,6 \mu \mathrm{m}$.
\end{abstract}

Abstract. - A calorimeter for the measurement of weak absorption coefficients is described. It is a conduction type calorimeter, the thermal powermeters used are semiconductor Peltier effect devices. This apparatus includes a correction for the light scattered in the sample. Its sensitivity is about $10^{-5} \mathrm{~cm}^{-1}$ and the accuracy better than $10 \%$ around $10^{-2} \mathrm{~cm}^{-1}$. Some results obtained at $10.6 \mu \mathrm{m}$ with $\mathrm{GaAs}, \mathrm{Ge}, \mathrm{NaCl}$ and $\mathrm{KBr}$ are given.

1. Introduction. - La méthode classique de détermination de l'absorption optique à partir des mesures de transmission et de réflexion ne permet pas d'atteindre des coefficients d'absorption inférieurs à environ $10^{-2} \mathrm{~cm}^{-1}$ pour des échantillons ayant une épaisseur de l'ordre du cm. Pour de nombreuses applications : fibres optiques, fenêtres des lasers à $\mathrm{CO}_{2}$ par exemple, il est nécessaire d'utiliser des matériaux dont les coefficients d'absorption aux longueurs d'onde utiles sont au-dessous de cette limite. En particulier, pour les fenêtres des lasers à $\mathrm{CO}_{2}$ de haute puissance, on recherche des matériaux ayant une absorption de l'ordre de $10^{-3} \mathrm{~cm}^{-1}$ à $10,6 \mu \mathrm{m}$.

Pour analyser ces matériaux, il est alors nécessaire de réaliser des mesures calorimétriques de l'absorption. Nous décrivons ici un appareil destiné à effectuer ces mesures en éliminant au maximum les effets parasites qui peuvent s'y introduire. Cet appareil, primitivement destiné à travailler à $10,6 \mu \mathrm{m}$, peut évidemment être utilisé à d'autres longueurs d'onde à condition de disposer d'une source monochromatique puissante (de l'ordre du watt).

Plusieurs appareillages de ce type ont été décrits dans la littérature [1-7]. Le processus le plus classique consiste à utiliser un calorimètre adiabatique qui contient l'échantillon sur lequel on envoie une impulsion laser transportant une énergie bien déterminée. On mesure l'échauffement de l'échantillon à l'aide d'un thermocouple. Un tel dispositif, simple dans son principe, présente plusieurs inconvénients : l'isolation thermique n'est jamais parfaite, ne serait-ce qu'à cause des

(*) Cette étude a été réalisée avec l'appui de la D. R. M. E. pertes dans les fils du thermocouple; le positionnement de l'échantillon est délicat ; la capacité calorifique des échantillons doit être bien connue; le thermocouple doit être parfaitement protégé du faisceau laser direct et des faisceaux diffusés, ce qui est souvent incompatible avec un bon contact thermique sur l'échantillon.

L'appareil décrit ici est un système à conduction qui permet d'éliminer pratiquement tous les inconvénients sans pour cela être d'une réalisation délicate.

2. Principe. - Le principe consiste à mesurer la puissance thermique absorbée par l'échantillon $\left(P_{\mathrm{a}}\right)$ lorsque celui-ci est traversé par un flux de puissance $\left(P_{\mathrm{i}}\right)$. Pour cela, la chaleur absorbée est évacuée vers la masse thermique à travers un élément qui joue le rôle de fluxmètre thermique, c'est-à-dire un système dont la réponse dépend de la puissance qui le traverse. L'adoption d'une méthode comparative permet d'éliminer toutes les causes d'erreur provenant des fuites thermiques.

Cependant, comme dans les systèmes mentionnés précédemment, une partie de la lumière peut être diffusée à l'intérieur ou à la surface de l'échantillon, puis atteindre les fluxmètres où elle sera transformée en chaleur puisque ceux-ci ne sont pas transparents. Cette lumière diffusée est alors comptée comme lumière absorbée, ce qui perturbe les mesures, surtout pour les faibles coefficients d'absorption. Afin de pouvoir tenir compte de cet effet, le calorimètre réalisé comporte un système de mesure de la lumière diffusée reçue par les fluxmètres.

La figure 1 montre le schéma de principe de l'appareil. On voit que quatre fluxmètres $F_{\mathrm{a}}, F_{\mathrm{a}}^{\prime}$ et $F_{\mathrm{d}}, F_{\mathrm{d}}^{\prime}$ 


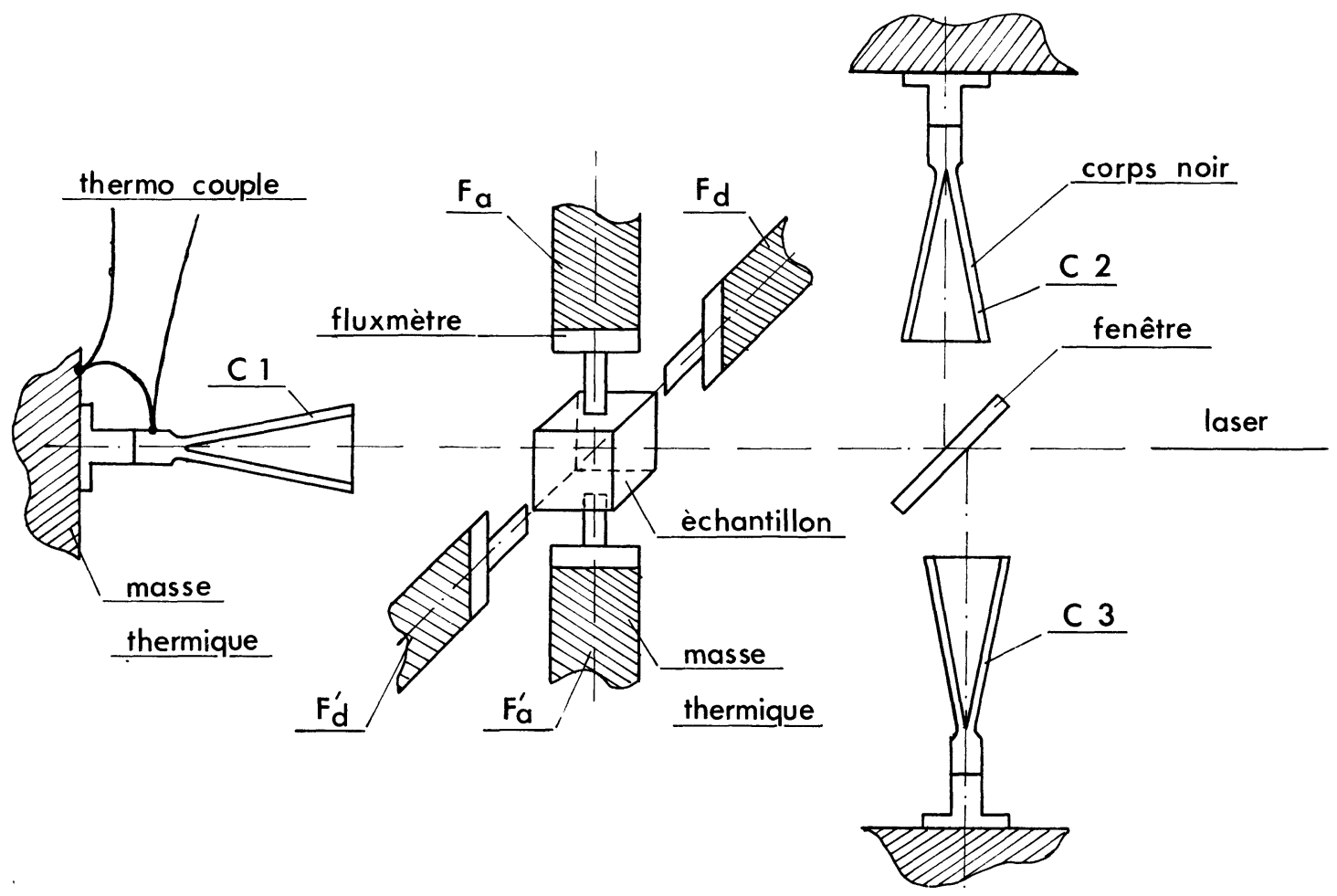

Fig. 1. - Schéma de principe de la mesure.

entourent l'échantillon d'une manière parfaitement symétrique. $F_{\mathrm{a}}$ et $F_{\mathrm{a}}^{\prime}$ diamétralement opposés, assurent le positionnement de l'échantillon et servent à la mesure de $P_{\text {abs }}$ qu'ils évacuent vers la masse thermique. $F_{\mathrm{d}}$ et $F_{\mathrm{d}}^{\prime}$ situés à $90^{\circ}$ des premiers, ne touchent pas l'échantillon, ils ne reçoivent donc que la puissance diffusée, ce qui permet sa mesure. Notons que les rôles respectifs des fluxmètres peuvent être inversés pour contrôler la symétrie du système. On a donc :

$$
P_{\text {abs }}=\left(F_{\mathrm{a}}+F_{\mathrm{a}}^{\prime}\right)-\left(F_{\mathrm{d}}+F_{\mathrm{d}}^{\prime}\right) .
$$

Les sommes et la différence peuvent être réalisées directement par le branchement des fluxmètres en série et en différentielle, ce qui élimine les dérives de l'ensemble.

La puissance du laser est mesurée à l'aide de deux cônes, placés l'un derrière l'échantillon $\left(\mathrm{C}_{1}\right)$ et l'autre perpendiculairement au faisceau à l'avant de l'échantillon $\left(\mathrm{C}_{3}\right)$. Ce dernier reçoit une partie constante du faisceau incident par l'intermédiaire d'un miroir semiréfléchissant. Sur son autre face, ce miroir renvoie une partie du faisceau réfléchi par la face avant de l'échantillon vers un troisième cône $\left(\mathrm{C}_{2}\right)$ qui permet donc une mesure du coefficient de réflexion de l'échantillon.

Tous ces cônes constituent des corps noirs et absorbent donc toute la puissance qu'ils reçoivent. Cette puissance est ensuite évacuée vers la masse thermique par l'intermédiaire d'une conductance thermique entre les extrémités de laquelle il apparaît une différence de température directement reliée à la conductance thermique et à la puissance évacuée.
3. Description de l'appareil. - La figure 2 montre le schéma général de l'appareil réalisé.

Sur le trajet du faisceau laser, il comprend :

- Deux séries de diaphragmes de $\varnothing 5 \mathrm{~mm}$, qui définissent l'orientation du faisceau et sa direction;

- Une lame à $45^{\circ}$ en GaAs (D) qui sert de miroir semi-réfléchissant et de fenêtre d'entrée du calorimètre proprement dit ;

- Le cube échantillon (E) dont les dimensions optimales sont $15 \times 15 \times 15 \mathrm{~mm}$;

- Le cône $\left(\mathrm{C}_{1}\right)$ de mesure de la lumière transmise avec sa résistance thermique (B).

Ce cône, comme les deux autres $\left(\mathrm{C}_{2}\right.$ et $\left.\mathrm{C}_{3}\right)$ a une ouverture de $20^{\circ}$, ce qui garantit un nombre minimal de 5 réflexions internes à tous les rayons du faisceau incident. De plus, ces cônes sont réalisés en cuivre poli et noirci. Sur chacun d'eux on note la présence d'une résistance chauffante $(\mathrm{G})$ qui sert à l'étalonnage de la résistance thermique du socle. Ces résistances sont laissées en place après étalonnage, de manière à ne pas modifier les pertes qu'elles peuvent entraîner. La capacité calorifique de chaque cône et la valeur de la résistance thermique ont été choisies pour réaliser un bon compromis entre la sensibilité de réponse et la constante de temps de la mesure. Celle-ci doit en effet être de l'ordre de grandeur de celle du système des fluxmètres de mesure de la puissance absorbée.

La différence de température entre les extrémités de chaque résistance thermique est mesurée à l'aide d'un thermocouple cuivre-constantan, dont la sensibilité est d'environ $40 \mu \mathrm{V} /{ }^{\circ} \mathrm{C}$. 


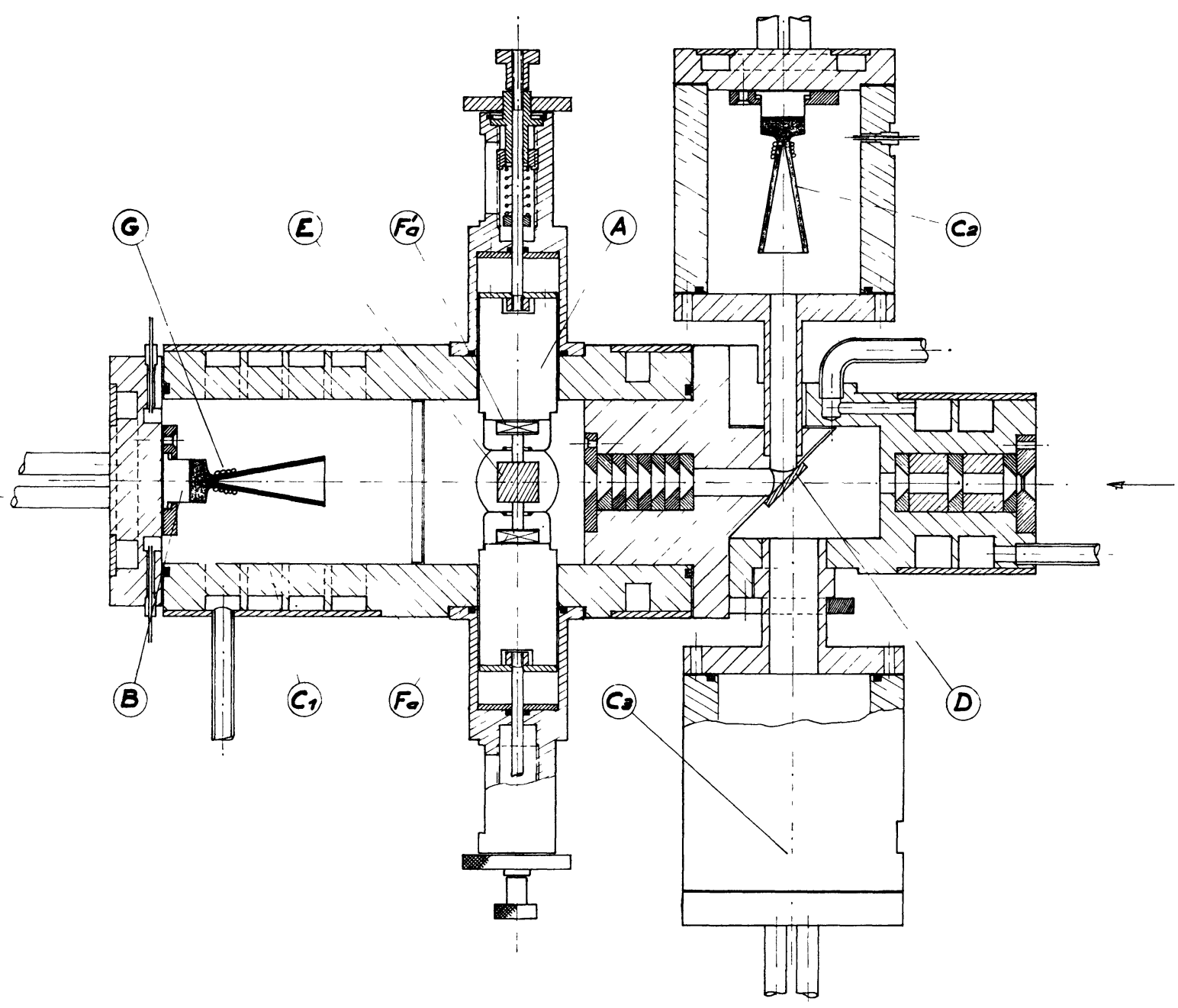

FIG. 2. - Schéma général du calorimètre monté.

Perpendiculairement au trajet du faisceau, on trouve :

- Les cônes $\left(\mathrm{C}_{2}\right)$ et $\left(\mathrm{C}_{3}\right)$ de mesure de la lumière incidente et réfléchie. Ils sont identiques à $\mathrm{C}_{1}$;

- Les systèmes de support des fluxmètres qui maintiennent l'échantillon en place par l'intermédiaire de deux capteurs de cuivre doré. La pression de ces doigts sur l'échantillon doit être suffisante pour assurer le contact thermique, sans toutefois détériorer les matériaux ; elle doit en outre être bien reproductible. Son ajustement s'effectue par le système du ressort visible sur la figure 2 .

- Les fluxmètres $F_{\mathrm{a}}$ et $F_{\mathrm{a}}^{\prime}$. Ils sont constitués par des éléments à effet Peltier (Cogie, réf. FC 1-4-71-10). Ces éléments, normalement destinés à pomper des calories lorsqu'ils sont traversés par un courant, sont ici utilisés en sens inverse. Ils sont équivalents à un ensemble de thermocouples, leurs éléments constituant la résistance thermique d'évacuation des calories vers la masse $\mathbf{A}$.

Ce dispositif a une sensibilité bien supérieure à un thermocouple classique, il est en outre plus facile d'emploi. Les blocs de cuivre A coulissent à l'intérieur du corps du calorimètre.
Autour des fluxmètres sont disposés des écrans qui éliminent la possibilité qu'une partie du faisceau laser ne les atteigne après des diffusions accidentelles.

Toujours pour éliminer ces diffusions, qui sont particulièrement critiques avec les faisceaux laser, compte tenu de leur puissance, l'ensemble du calorimètre a été noirci. En outre, comme il est nécessaire que le corps du calorimètre soit isotherme, toutes les pièces sont réalisées en cuivre et on a veillé à ce que les contacts thermiques entre chacune, en particulier entre les blocs mobiles A et le corps fixe, soient bons. De plus, l'évacuation de la puissance totale reçue par l'ensemble et sa stabilisation en température sont assurées par une cir. culation de liquide thermostatée dans toutes les pièces fixes. Le thermostat utilisé (Lauda K $4 \mathrm{R}$ ) peut dissiper $250 \mathrm{~W}$ et stabilise la température à $\pm 0,02{ }^{\circ} \mathrm{C}$ près.

Enfin, à l'intérieur du calorimètre, on réalise un vide de $10^{-3}$ torr environ pour éliminer les pertes par convection. Seules subsistent donc les pertes par rayonnement, mais, d'une part elles sont très faibles puisqu'on travaille dans des conditions quasi isothermes au niveau de l'échantillon et, d'autre part, comme nous le verrons plus loin, l'étalonnage tient compte d'un éventuel effet de ce type. 
La source de rayonnement utilisée pour l'application envisagée est un laser à $\mathrm{CO}_{2}$ (Philips, réf. PW 9960) délivrant une puissance de $30 \mathrm{~W}$ environ avec un diamètre de faisceau de $1 \mathrm{~cm}$ et une divergence de $4 \times 10^{-3}$ radian. Au niveau de l'échantillon, la puissance utile est de $2 \mathrm{~W}$.

4. Détermination du coefficient d'absorption. - Deux mesures sont nécessaires : celle de la puissance du faisceau et celle de la puissance absorbée. Pour déterminer le coefficient d'absorption, nous avons utilisé deux méthodes indépendantes, afin d'éliminer d'éventuelles causes d'erreurs systématiques.

4.1 Mesure DE LA PUISSANCE DU FAISCEAU. Si $1 / k$ est la résistance thermique placée derrière un cône, on mesure aux extrémités de cette résistance une différence de température $\Delta T$ reliée à la puissance reçue par

$$
\Delta T=1 / k \cdot P \text { reçue } .
$$

La figure 3 représente les courbes de réponse $e$ des thermocouples, tracées pour chaque cône à l'aide des résistances chauffantes $(G)$. On constate que la linéarité est bonne, ce qui permet de définir une sensibilité :

$$
\alpha=e / p \text {. }
$$

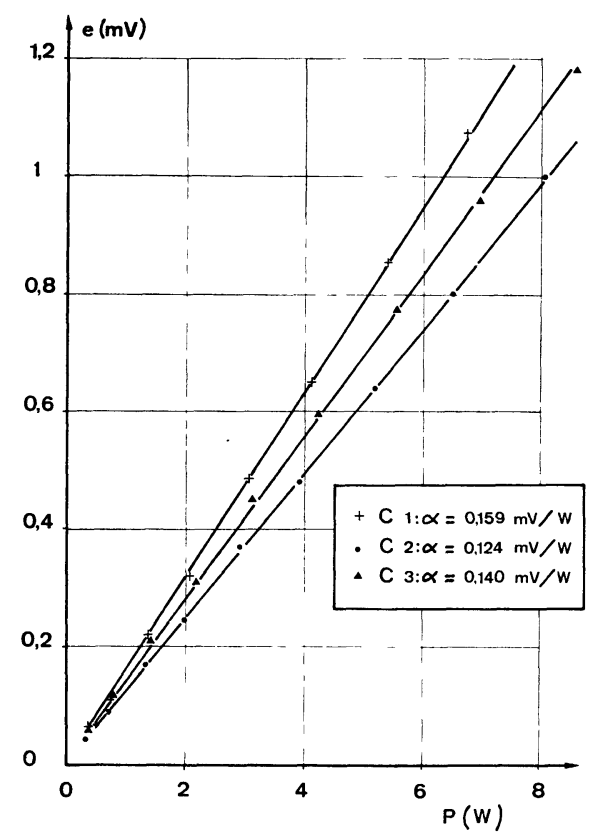

Fig. 3. - Courbes d'étalonnage des cônes de mesure des puissances incidentes, transmises et réfléchies. $e:$ réponse de thermocouple, $P$ : puissance reçue.

4. 2 Première MÉTHOde DE DÉTERMINATION DU COEFFICIENT D'ABSORPTION $\beta$. - Elle consiste à étalonner directement les fluxmètres en puissance. Pour cela, on remplace l'échantillon par une résistance dans laquelle on dissipe une puissance Joule mesurable.

Afin de se trouver dans des conditions expérimentales strictement identiques à celles des mesures, ce qui garantit que l'on tient compte d'éventuelles pertes, nous avons utilisé comme résistance un cube de silicium de dimensions identiques à celles de l'échantillon, sur deux faces duquel ont été réalisés des contacts ohmiques. Le courant est amené par les doigts assurant le contact thermique, ce qui évite d'ajouter des fils.

La figure 4 montre les courbes $v(P)$ ainsi obtenues. On a contrôlé l'égalité des réponses de $F_{\mathrm{a}}$ et de $F_{\mathrm{a}}^{\prime}$. On constate que les réponses ne sont pas linéaires sur les trois décades considérées, ce qui nécessite d'utiliser directement cette courbe pour la détermination de la puissance absorbée.

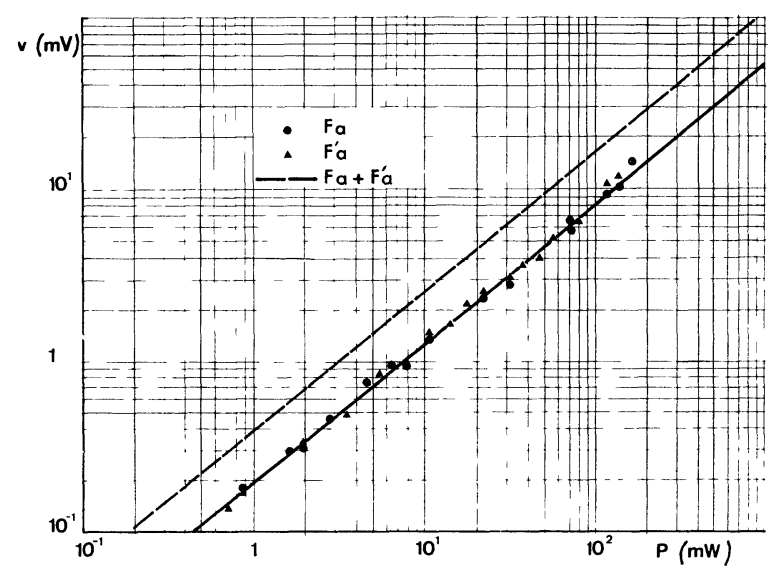

Fig. 4. - Courbes d'étalonnage en puissance des fluxmètres de mesure de la puissance absorbée $F_{\mathrm{a}}$ et $F_{\mathrm{a}}^{\prime} . v$ : réponse des éléments Peltier, $P$ : puissance dissipée.

Le même étalonnage est réalisé pour les fluxmètres $F_{\mathrm{d}}$ et $F_{\mathrm{d}}^{\prime}$.

Pour la mesure de l'absorption, l'échantillon à étudier, poli sur ses 6 faces, est mis en place ; on envoie le faisceau laser, à l'équilibre on note :

$$
P_{\mathrm{abs}}=\left(F_{\mathrm{a}}+F_{\mathrm{a}}^{\prime}\right)-\left(F_{\mathrm{d}}+F_{\mathrm{d}}^{\prime}\right)
$$

et $P_{0}$, la puissance transmise.

Cette dernière est en effet suffisante pour déterminer le coefficient d'absorption qui prend la forme [1]:

$$
\beta=\frac{1}{x} \frac{P_{\mathrm{abs}}}{P_{0}} \cdot \frac{2 n}{1+n^{2}}
$$

où $x$ est l'épaisseur de l'échantillon, et $n$ son indice. Cette formule n'est valable que pour $P_{\text {abs }} \ll P_{0}$, ce qui est toujours vrai pour les échantillons auxquels on s'intéresse ici.

\section{3 SECONDE MÉTHODE POUR LA DÉTERMINATION} DU COEFFICIENT D'ABSORPTION $\beta$. - On dispose, dans un premier temps, à la place de l'échantillon, un cube métallique dans lequel on a noyé un thermocouple. Ce cube est chauffé par un processus quelconque : par exemple directement à l'aide du laser. On enregistre alors la réponse des Peltiers en fonction de celle du thermocouple. Le refroidissement est suffisamment lent pour que l'équilibre thermique soit bon. 
La figure 5 représente la courbe $v^{\prime}\left(T-T_{0}\right)$ ainsi obtenue. On constate sa parfaite linéarité.

Pour connaître la puissance absorbée, il faut encore déterminer la conductance thermique $K$ des fluxmètres.

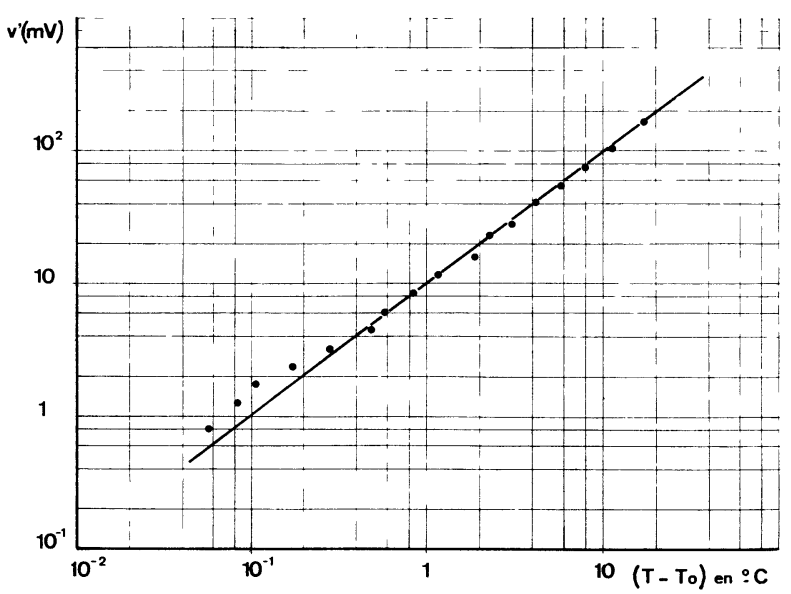

FIG. 5. - Courbes d'étalonnage en température des fluxmètres $v^{\prime}$ : réponse des éléments Peltier. $\left(T-T_{0}\right)$ : différence de température entre l'échantillon et la masse thermique.

Ceci s'effectue en mesurant les constantes de temps de décroissance des réponses des fluxmètres en présence d'un échantillon de capacité calorifique et de masse bien connues. En effet, au refroidissement, la température évolue suivant la loi :

$$
T(t)-T_{0}=\left(T_{1}-T_{0}\right) \cdot \exp \left(-\frac{t}{\tau}\right)
$$

avec

$$
\tau=J \frac{\sum m c}{K}
$$

$\sum m c$ représente la capacité calorifique de la partie chaude, $K$ la conductance thermique des fluxmètres, $T_{1}$ la température à $t=0$ et $T_{0}$ la température de la masse. La mesure de $\tau$ est effectuée avec différents matériaux. $\left(\sum m c\right)$ étant facilement déterminé, on en déduit $K$.

La puissance absorbée pour une réponse $v^{\prime}$ des Peltiers s'écrit alors :

$$
P_{\mathrm{abs}}=K\left(T_{\mathrm{e}}-T_{0}\right)
$$

$\left(T_{\mathrm{e}}-T_{0}\right)$ est déterminé à l'équilibre à l'aide de la courbe d'étalonnage de la figure 5 . On passe au coefficient d'absorption par la même formule que précédemment.

4.4 COMParaison Des Résultats : PRÉCision, SENSIBILITÉ. - Afin de déterminer la précision de la mesure de $\beta$, nous avons effectué une série d'essais par chaque méthode sur un même échantillon de GaAs semi-isolant (dopé au chrome). Nous avons obtenu les valeurs moyennes suivantes :

$$
\begin{aligned}
& 1^{\text {re }} \text { méthode }: \beta=1,25 \times 10^{-2} \mathrm{~cm}^{-1} \\
& 2^{\mathrm{e}} \text { méthode }: \beta=1,26 \times 10^{-2} \mathrm{~cm}^{-1} .
\end{aligned}
$$

Pour une mesure unique, l'incertitude définie par l'écart maximal relevé au cours d'une série de mesures sur un même échantillon dans des conditions expérimentales différentes, est de :

$$
\begin{aligned}
& 1^{\mathrm{re}} \text { méthode }: \pm 0,1 \times 10^{-2} \mathrm{~cm}^{-1} \\
& 2^{\mathrm{e}} \text { méthode }: \pm 0,2 \times 10^{-2} \mathrm{~cm}^{-1} .
\end{aligned}
$$

On constate que la deuxième méthode conduit à une dispersion supérieure des valeurs expérimentales, ce qui semble normal, du fait qu'elle est moins directe que la première. La sensibilité de la mesure est de quelque $10^{-5} \mathrm{~cm}^{-1}$ pour des échantillons de $15 \mathrm{~mm}$ d'épaisseur et une puissance $P_{0}$ sur l'échantillon de $2 \mathrm{~W}$ environ.

Notons que la correction de lumière diffusée est importante. Pour des monocristaux de GaAs ayant subi un polissage optique, elle atteint $10 \%$. Si on tient compte de l'angle solide des capteurs, cela correspond à une importante perte par diffusion.

L'importance de cette lumière diffusée peut s'expliquer par la présence dans ces matériaux de microprécipités tels que ceux observés par Van der Sande en microscopie électronique [8], ou par des inhomogénéités affectant l'indice du semiconducteur. Ce dernier mécanisme doit être rapproché de l'existence de queues de bande importantes dans ces matériaux [9].

Enfin, la mesure des constantes de temps fournit une détermination de la capacité calorifique des matériaux étudiés. Pour GaAs, nous avons trouvé : $C=0,1 \mathrm{cal} \cdot \mathrm{g}^{-1} \cdot{ }^{\circ} \mathrm{C}^{-1}$. Les valeurs trouvées dans la littérature sont 0,064 [1] et 0,084 [10].

Conclusion. - A l'aide de cet appareil, nous avons mesuré l'absorption dans une série de monocristaux de GaAs. Les coefficients $\beta$ trouvés vont de $0,85 \times 10^{-2} \mathrm{~cm}^{-1}$ à $1,25 \times 10^{-2} \mathrm{~cm}^{-1}$, sauf un échantillon qui fournit $2 \times 10^{-2} \mathrm{~cm}^{-1}$. Nous avons regroupé dans le tableau suivant, à titre de comparaison, les valeurs rapportées dans la littérature :

$\begin{array}{lc}\text { Auteur [réf.] } & \beta \text { en } 10^{-2} \mathrm{~cm}^{-1} \\ \text { Horrigan [1] } & - \\ \text { Thomson [4] } & 1,7 \\ \text { Comly [11] } & 0,5 \text { à } 3 \\ \text { Bendow [2] } & 0,6 \\ \text { Weil [12] } & 1,5 \\ \text { Haggerty [13] } & 1,2 \\ \text { Présente étude } & 1 \text { à } 1,25 \\ & 0,9 \text { à } 1,25\end{array}$

Nous avons également étudié des cristaux de $\mathrm{Ge}$, de $\mathrm{NaCl}$ et de $\mathrm{KBr}$; les résultats sont les suivants :

$$
\begin{aligned}
& \mathrm{Ge}: 1,6 \times 10^{-2} \mathrm{~cm}^{-1} \\
& \mathrm{NaCl}: 0,2 \times 10^{-2} \mathrm{~cm}^{-1} \\
& \mathrm{KBr}: 0,27 \times 10^{-2} \mathrm{~cm}^{-1} .
\end{aligned}
$$

L'importance de la correction de lumière diffusée augmente évidemment lorsque les coefficients d'absorp- 
tion diminuent, elle devient alors absolument nécessaire. De même, dans le cas de matériaux polycristallin ou inhomogène, cette correction peut devenir très importante.

Notons qu'avec une petite modification du système de fixation des échantillons, il est possible d'effectuer des mesures sur des plaquettes.

En conclusion, l'appareil décrit ici permet d'effectuer des mesures absolues du coefficient d'absorption avec un faible risque d'erreurs systématiques. Le positionnement automatique de l'échantillon et sa reproductibilité autorisent la réalisation de mesures systématiques en grand nombre dans un minimum de temps.

Remerciements. - Nous remercions MM. P. Parquet et J. P. Farges de l'aide technique qu'ils nous ont apportée pour la construction de cet appareil et la réalisation des mesures.

\section{Bibliographie}

[1] Horrigan, F. et RudKo, R. I., Rapport Raytheon Research Div., 1969.

[2] Bendow, R., Hordvik, A., Lipson, H. et Skolnik, L., AFCRL Technical report 72-0404, June 1972.

[3] Hass, M., Patton, F. W., Nav. Res. Lab. report, jan. 1972.

[4] Thompson, A. G., J. Electron. Mater. 2 (1973) 47.

[5] SMITH, R. L., IEEE Trans. Instrum. Meas. 21 (1972) 434.

[6] Hass, M., Davisson, Z. W., Rosenstock, H. B., BabisKIA, J., Appl. Opt. 14 (1975) 1128.

[7] Nurmikкo, A. V., Appl. Phys. Lett. 26 (1975) 175.
[8] Van der Sande, J. B., Peters, E. T., J. Appl. Phys. 45 (1974) 1297.

[9] Leyral, P., Bois, D., Pinard, P., à paraître dans Phys. stat. sol.

[10] HieKey, J. E., Electronic Ind., Feb. 1963.

[11] Comly, J., Garmire, E. et Yariv, A., J. Appl. Phys. 38 (1967) 4091

[12] WeIL, R., J. Appl. Phys. 40 (1969) 2857.

[13] Haggerty, J. S., Peters, F. T., Report on characterization of IR windows, 1972.

[14] Hicks, H. G. B., Hanley, D. F., Solid State Commun. 7 (1969) 1463 